\title{
First-Order Insulator-to-Metal Mott Transition in the Paramagnetic 3D System $\mathrm{GaTa}_{4} \mathrm{Se}_{8}$
}

\author{
A. Camjayi, ${ }^{1}$ C. Acha,${ }^{1}$ R. Weht,${ }^{2,3}$ M. G. Rodríguez, ${ }^{1}$ B. Corraze,${ }^{4}$ E. Janod,${ }^{4}$ L. Cario, ${ }^{4}$ and M. J. Rozenberg ${ }^{5}, *$ \\ ${ }^{1}$ Departamento de Física, FCEN, UBA, and IFIBA, CONICET, \\ Pab. 1, Ciudad Universitaria, 1428 Buenos Aires, Argentina. \\ ${ }^{2}$ Gerencia de Investigación y Aplicaciones, Comisión Nacional de Energía Atómica (CNEA). \\ Avenida General Paz y Constituyentes, 1650 San Martín, Argentina. \\ ${ }^{3}$ Instituto Sábato, Universidad Nacional de San Martín-CNEA, 1650 San Martín, Argentina. \\ ${ }^{4}$ Institut des Matériaux Jean Rouxel (IMN), Université de Nantes, CNRS, \\ 2 rue de la Houssinière, BP32229, 44322 Nantes, France \\ ${ }^{5}$ Laboratoire de Physique des Solides, CNRS-UMR8502, Université de Paris-Sud, Orsay 91405, France.
}

\begin{abstract}
The nature of the Mott transition in the absence of any symmetry braking remains a matter of debate. We study the correlation-driven insulator-to-metal transition in the prototypical 3D Mott system $\mathrm{GaTa}_{4} \mathrm{Se}_{8}$, as a function of temperature and applied pressure. We report novel experiments on single crystals, which demonstrate that the transition is of first order and follows from the coexistence of two states, one insulating and one metallic, that we toggle with a small bias current. We provide support for our findings by contrasting the experimental data with calculations that combine local density approximation with dynamical mean-field theory, which are in very good agreement.
\end{abstract}

The Mott insulator is a basic notion in modern condensed matter physics, at the center of strongly correlated phenomena. In particular, the correlation-driven Mott metal-insulator transition takes place in systems with partially filled bands and in the absence of magnetic order or any other type of symmetry breaking 1 . This transition is possibly the simplest nontrivial strong correlation effect in a lattice system and can be considered the electronic analogue of the familiar water-vapor transition. The Hubbard model is its minimal theoretical realization, and its solution within dynamical mean-field theory (DMFT), in the 1990s, provided a detailed scenario of the transition [2]. One of the key predictions is that it is of first order with an associated region of two competing coexistent solutions: a Mott insulator and a correlated metal 2. Their relative stability depends on the strength of correlations, which may be physically tuned by, for instance, externally applied pressure. One of the best candidates to exhibit the insulator-to-metal transition (IMT) was the compound $\mathrm{V}_{2} \mathrm{O}_{3}$, which has been continuously studied since the 1970s [3].

While many features of the IMT in $\mathrm{V}_{2} \mathrm{O}_{3}$ do resemble the DMFT scenario 446, the comparison of the experimental data with the theory is not straightforward. In fact, density functional calculations using the local density approximation (LDA) of $\mathrm{V}_{2} \mathrm{O}_{3}$ show that a multitude of bands cross the Fermi energy, rendering the connection to a simple Hubbard model physics a matter of ongoing debate [7. For instance, the orbital degrees of freedom seem to be active across the metal-insulator transition leading to an orbitally ordered state [8]. Moreover, the paramagnetic Mott transition does not occur for the stoichiometric compound, and a small chemical substitution of $\mathrm{V}$ by $\mathrm{Cr}$ is required 3. This poses further concerns on the validity of the single occupation constraint and on the eventual interplay of correlations with disorder. Therefore, despite the extensive experimental work on $\mathrm{V}_{2} \mathrm{O}_{3}$, which is certainly a fascinating system, the detailed scenario for the 3D Mott-Hubbard transition remains unclear [7.

Here, we present a new approach to this long-standing problem and provide compelling experimental and theoretical evidence of the nature of the quantum IMT in the Mott system $\mathrm{GaTa}_{4} \mathrm{Se}_{8}$ (GTS), which is a far simpler compound than $\mathrm{V}_{2} \mathrm{O}_{3}$. We performed novel transport experiments on single crystals across the IMT as function of temperature and quasihydrostatic pressure. We find that the transition is of first order due to the coexistence of two strongly correlated states, one insulating and one metallic. More specifically, within the transition region, where the resistivity shows nonmonotonic behavior, we show that we may reversibly commute between the two well defined states, by means of a small bias current. We further substantiate the interpretation of the experimental data with a theoretical study of the electronic properties by means of LDA+DMFT numerical calculations 9. We find our results in very good agreement with the present and previously reported experimental data, which provides strong support to the DMFT MottHubbard transition scenario.

GTS is a member of the family of chalcogenide compounds $\mathrm{AM}_{4} \mathrm{Q}_{8}$, with $\mathrm{A}=\{\mathrm{Ga}, \mathrm{Ge}\}, \mathrm{M}=\{\mathrm{V}, \mathrm{Nb}, \mathrm{Ta}$, $\mathrm{Mo}\}, \mathrm{Q}=\{\mathrm{S}, \mathrm{Se}, \mathrm{Te}\}$ [10 14, which were all classified as Mott insulators since they were expected to be metals from band structure calculations. The importance of strong correlations was further underlined by the re- 
port of a pressure-driven metal-insulator crossover in GTS along with indications of superconductivity above 11.5 GPa [10, 15] and of a doping-driven transition [16]. Furthermore, an intriguing electric-field-driven resistive switching effect, both volatile [14 and nonvolatile [17, was found in many members of the family. The mechanism proposed for that resistive switching transition [18, 19] was associated with Mott physics.

Unlike $\mathrm{V}_{2} \mathrm{O}_{3}$, these compounds have a rather simple structure. They are lacunar spinels with an fcc general symmetry [20, 21]. The active electrons are in the $\mathrm{M}_{4}$ clusters, which are themselves forming an fcc sublattice. Therefore, their molecular orbitals give rise to $t_{2 g}$-like conduction bands. The electron count in GTS indicates that these bands should contain a single unpaired electron per $\mathrm{Ta}_{4}$ cluster $[10$. The relatively large intercluster distance results in a small hopping amplitude and, hence, narrow partially filled conduction bands. All these features are, in fact, borne out from band structure calculations [11, 12, 22, 23, which display three $\mathrm{t}_{2 g}$ bands crossing the Fermi energy. However, as we mentioned before, all these systems turn out to be insulators. Significantly, GTS is a paramagnetic insulator and does not order down to the lowest measured temperatures [12, 24, which is possibly due to the magnetic frustration of the fcc arrangement of $\mathrm{Ta}_{4}$ clusters. Therefore, the GTS system is relatively simple and well suited to investigate the correlation-driven Mott transition.

In Fig. 1. we show experimental data of the temperature dependent resistivity $\rho(T)$ of GTS under various quasihydrostatic pressures $P$, from 1 to $5 \mathrm{GPa}$. The data show a huge resistivity change of 10 orders of magnitude at low temperature, as the system undergoes a dramatic insulator-metal transition. At low pressures, the system is a Mott insulator, and at high pressures, the state collapses to a correlated metal with anomalous resistivity. In fact, our measurements of $\rho(T)$ on single crystals unveil two key novel features with respect to previously published data obtained in polycrystalline samples [10]. Significantly, we observe (i) a nonmonotonic behavior in the metallic high-pressure phase $(\sim 5 \mathrm{GPa})$ and (ii) a strong hysteresis effect at the critical pressure region of the Mott transition $(\sim 3.5 \mathrm{GPa})$.

The nonmonotonic behavior of $\rho(T)$ is an indication of a strong correlation effect. In fact, it appeared in early theoretical studies of the single band Hubbard model within DMFT 2, 4. The experimental data show that at low $T$, the system has good metallic resistivity, which increases with $T$. However, around $100 \mathrm{~K}$, which is merely $\sim 0.01 \mathrm{eV}$ and almost 2 orders of magnitude less than the LDA bandwidth $\sim 0.7 \mathrm{eV}$ [23], the resistivity suddenly flattens out and turns semiconducting at higher temperatures.

Significantly, at intermediate pressures $(P=3.5 \mathrm{GPa})$, within the IMT region, $\rho(T)$ shows strong hysteresis upon cooling and heating. The pressure range of the observed

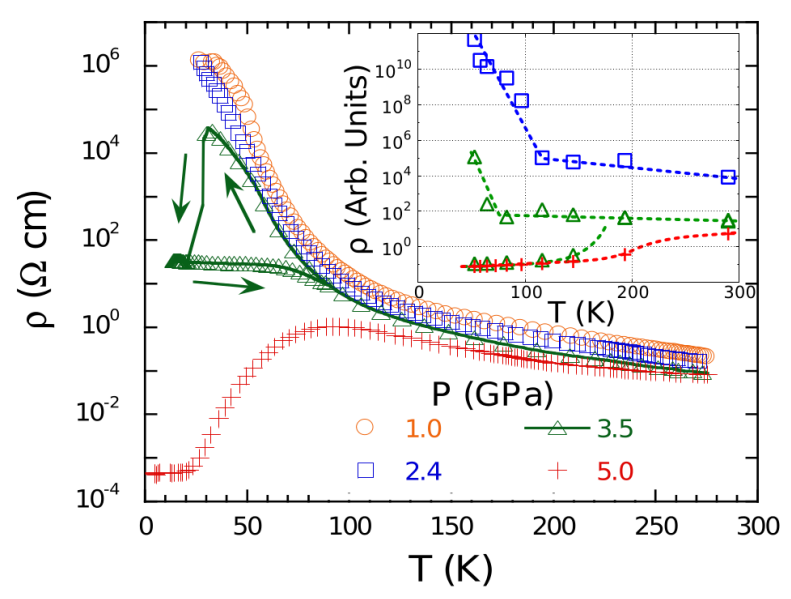

FIG. 1. Main panel: Resistivity as a function of temperature (4 K $\leq T \leq 300 \mathrm{~K}$ ) for pressures from 1 to $5 \mathrm{GPa}$ measured in a $\mathrm{GaTa}_{4} \mathrm{Se}_{8}$ single crystal (sample A). The data show insulating behavior at low $P$ (orange circles and blue squares), metallic behavior at high $P$ (red crosses), and strong hysteresis behavior at low $T \lesssim 80 \mathrm{~K}$ on the metal-insulator transition region (green triangles). Inset: $\mathrm{LDA}+\mathrm{DMFT}$ results for the resistivity as a function of the temperature. The metal (red crosses), insulator (blue squares), and coexistent (green triangles) solutions were obtained at $U=1,1.2$, and $1.1 \mathrm{eV}$, respectively. The lines are a guide for the eye.

hysteresis effects in different samples (see also Fig. 2 ) is rather narrow and therefore easy to miss and challenging to observe. This hysteresis effect is an unambiguous indication of the first-order character of the transition.

We now turn the focus on the resistivity within the hysteresis region. One of the main findings is that the observed hysteresis is not an ordinary memory effect, but it is rather due to the presence of two coexistent quantum states, one continuously connected to the Mott insulator and the other to the correlated metal. In Fig. 2 we show the strong hysteresis effects that are observed in the transition region. The square (red) data points were obtained cooling down the system and the triangles (black and purple) and circles (green) correspond to heating. While cooling, the applied bias current was systematically decreased, from $10 \mu \mathrm{A}$ at $300 \mathrm{~K}$ down to $0.02 \mu \mathrm{A}$ at $4 \mathrm{~K}$, to prevent spurious Joule heating effects. Through the hysteresis region $(\sim 60$ to $25 \mathrm{~K})$ the bias current was $0.1 \mu \mathrm{A}$. Along the cooling process the system simply remains on the insulator state down to the lowest temperature. However, the activated behavior shows a smaller gap $(\sim 0.11 \mathrm{eV})$ with respect to the lower pressure data $(\sim 0.12 \mathrm{eV}$ at $2.4 \mathrm{GPa}$ and $\sim 0.24 \mathrm{eV}$ at ambient pressure [13]), indicating a further reduction of the correlation gap. The interesting resistive bistability effect is observed upon heating. We started to heat up applying a bias current of $0.1 \mu \mathrm{A}$. Initially, the data follow essentially the same insulator-state curve obtained during the cooling process. However, at $28 \mathrm{~K}$, there is 


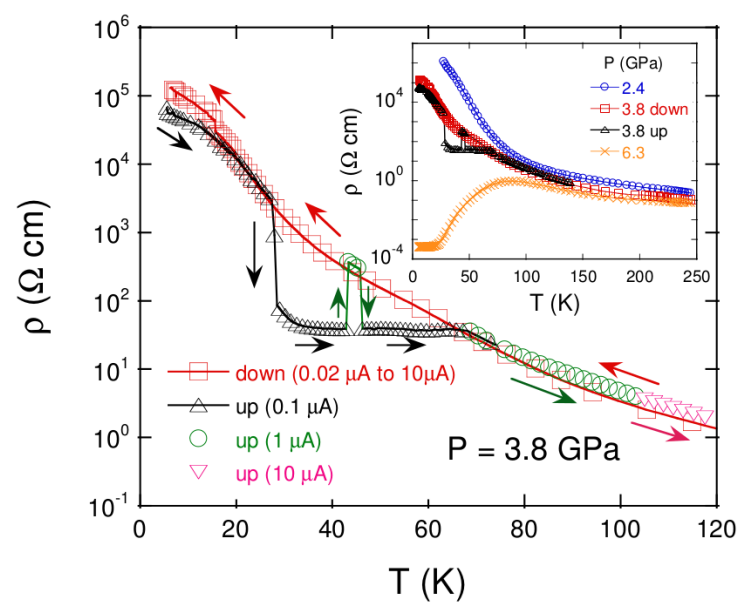

FIG. 2. Resistivity of a $\mathrm{GaTa}_{4} \mathrm{Se}_{8}$ single crystal (sample B) measured within the coexistence region as a function of temperature at $3.8 \mathrm{GPa}$. The commutation metal insulator in the heating process is controlled by a small bias current (see the text). Inset: Overall behavior of resistivity as a function of temperature for different pressures (2.4 GPa for sample A and 3.8 and $6 \mathrm{GPa}$ for sample $\mathrm{B}$ ).

a sudden decrease of the resistance of almost 2 orders of magnitude. Upon further heating, $\rho(T)$ remains flat with no activated behavior, indicating the onset of a (bad) metallic state. At $43 \mathrm{~K}$, we increased the bias current to $1 \mu \mathrm{A}$ to induce a small Joule heating and observed a sudden jump of the resistance, precisely to the insulatorstate curve. We heated up the system further up, to $46 \mathrm{~K}$, and it followed the insulator-state data. At that point, we decreased the bias current back to $0.1 \mu \mathrm{A}$ and observed that the resistance immediately switched back to the metallic state curve. Upon further heating the system remained in the metallic state. At about $70 \mathrm{~K}$, the resistivity of the heating curve merged with the cooling one, and from there on, no more hysteresis is observed.

The experiments that we just described clearly demonstrate the presence of two coexistent states, a Mott insulator and a correlated metal, which originate the first-order insulator-to-metal transition. This scenario is in qualitative agreement with the early studies of the Mott transition in the single band Hubbard model within DMFT, done in the early 1990s. Since then, the DMFT method has evolved from the original model Hamiltonian studies to incorporate the realistic electronic structure of different material compounds, which is provided by density functional methods [9, 25]. This development enables us to perform theoretical studies of correlation effects that are specific to each compound. Thus, we shall provide further support to the first-order IMT scenario by means of an LDA+DMFT numerical study using the realistic electronic structure of GTS 23. Details of the numerical methods are provided in the Supplemental Material [26].
An important issue of the LDA+DMFT method is the determination of the magnitude of the correlation parameter $U$, originated in the local Coulomb interactions. Its $a b$ initio determination remains a challenging problem. Here, we shall use a physical argument to estimate its value. For simplicity, we shall also assume a single $U$ value for all interorbital repulsion parameters and we neglect the Hund's interaction. From experiments we know that GTS is, in fact, a Mott insulator; hence, the parameter $U$ must be large enough to destroy the LDA metallic state. However, a second experimental fact is that under the effect of external pressure, GTS undergoes an insulator-to-metal transition. External pressure produces an increase of the bandwidth $W$ of about 0.023 $\mathrm{eV} / \mathrm{GPa}$ [15. It thus decreases the strength of the correlation effects $U / W$ (since $U$ is rather independent of pressure) and leads to the onset of the IMT in GTS. Therefore, from these observations, we may conclude that the value of interaction $U$ should be such that places the system on the insulator side and near the IMT. Therefore, our strategy will be to first estimate the value of interaction $U$ that destroys the LDA metal and then to study the behavior of the system in the neighborhood of the transition to compare with the experiments. For simplicity, we shall use $U$ as a parameter and keep the LDA band structure fixed, since the conclusions of our study do not depend on this choice (see the Supplemental Material [26] for details).

In Fig. 3(a), we show the LDA+DMFT results for the electron occupation versus chemical potential as a function of the local Coulomb repulsion $U$. We observe that for strong enough interaction, the $n$ vs. $\mu$ curves develop a plateau at $n=1$, which corresponds to the filling of GTS (i.e., one electron per $\mathrm{Ta}_{4}$ tetrahedron). The plateau denote the destruction of the metal and the onset of the incompressible Mott insulator state [36. From the data, we estimate that the GTS system at ambient pressure has a value of $U \approx 1.2 \mathrm{eV}$.
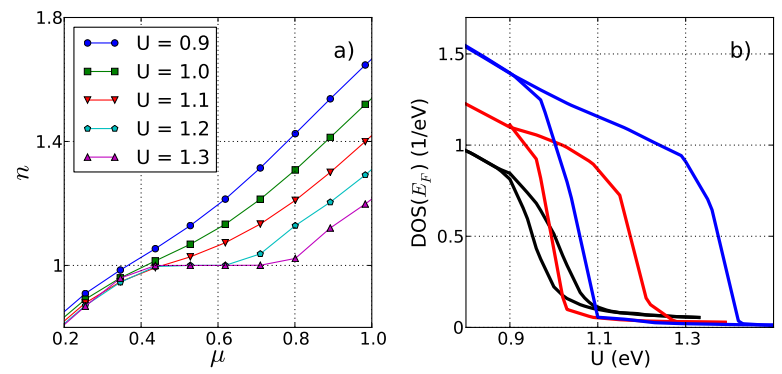

FIG. 3. (a) LDA+DMFT results of the occupation of the $t_{2 g}$ bands of GTS as a function of the chemical potential $\mu$ and the interaction $U$. The data show that the Mott gap opens at $n=1$ for $U \approx 1.2 \mathrm{eV}$. (b) Density of states at the Fermi energy as a function of $U$ for $n=1$ at $T=58,116$ and $232 \mathrm{~K}$ (top to bottom), indicating the coexistence of solutions. 
We now explore the neighborhood of the IMT to search for coexistent solutions and hysteresis. To numerically search for coexistent solutions, we simply "follow" one solution, say, the insulating one, and slowly lower the value of $U$ (i.e., the $U / W$ ratio) recomputing the converged solution at every step. Eventually, the solution has a sudden change to a qualitatively different state, i.e. the metallic solution. Then, we follow the same procedure for the metallic solution, now increasing the value of $U$, until we observe a sudden change. The region of $U$ values where two converged solutions are found determines the coexistent region. In Fig. 3(b) we show the hysteresis of the density of states (DOS) at the Fermi energy, which clearly distinguishes the insulator from the metal state. The coexistence region extends in a small window of $U / W$ values, narrows as the temperature is increased, and eventually disappears above $T \approx 230 \mathrm{~K}$. This is consistent with our experimental results, which showed two coexistent states within a narrow range of pressures, below $T \approx 100 \mathrm{~K}$. This agreement is remarkable, since it should be kept in mind that this temperature is quite a small energy scale, almost 2 orders of magnitude smaller than the LDA bandwidth $W$ and the interaction $U$, which emerges from a many-body effect. Within LDA+DMFT, we may also compute the resistivity $\rho(T)$ of the system. The results are shown in the inset of Fig. 1, which show a good agreement with the experimental results. The numerical data capture the basic qualitative features, namely, the relatively weak variation of $\rho$ at higher $T$, the many orders of magnitude of difference between the metallic and insulating resistivities at low $T$, and the nonmonotonic behavior of the correlated metal state.

Finally, we turn to the spectral functions, which reveal some unexpected features in regard to recent experiments [15]. The interacting band structure, as expected, displays a strong renormalization with respect to the LDA calculations. The results are shown in Fig. 4. For reference, we also display in the figure the LDA bands. The Mott insulator has lower and upper Hubbard bands, which are split by energy $\sim U$. They are very incoherent but still bear resemblance to the LDA dispersion. In the figure, we also show the local $\operatorname{DOS}(\omega)$. Interestingly, our results enable us to interpret and understand the apparent inconsistency between our presently estimated value of $U=1.2 \mathrm{eV}$ and the smaller one $\sim 0.55$ $\mathrm{eV}$ reported in a recent optical conductivity study of GTS [15]. Our numerical calculation reveals the existence of a secondary structure within the upper Hubbard band, a narrow subband at $\omega \sim 0.2 \mathrm{eV}$. This feature should lead to the presence of two distinct contributions in the optical conductivity spectrum, as indicated in Fig. 4 a more prominent one of magnitude $\omega \sim 1.2 \mathrm{eV}$ and a smaller one at $\sim 0.6 \mathrm{eV}$. This latter one is consistent with the value of the excitation reported in Ref. 15. The origin of this contribution can be traced to a multiorbital effect,

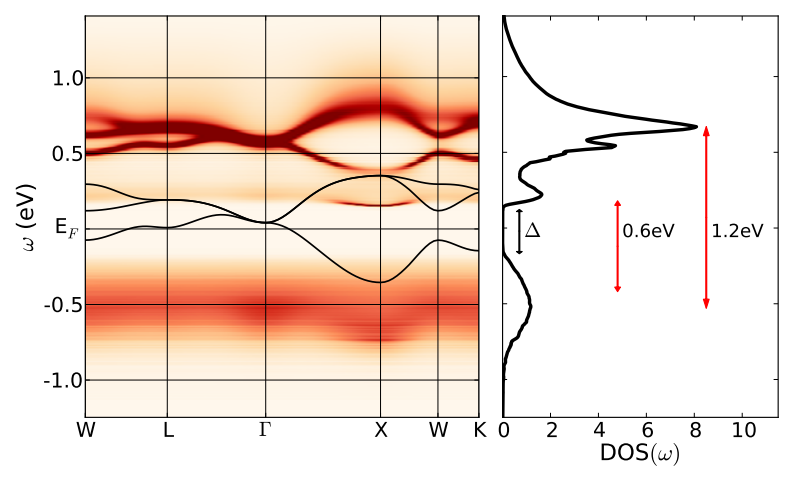

FIG. 4. LDA+DMFT calculation of the Mott insulator state. Left panel: Intensity plot of the electronic structure of the $\mathrm{t}_{2 g}$ bands of GTS obtained from calculations for $U=1.2 \mathrm{eV}$ and $T=58 \mathrm{~K}$. Note within the upper Hubbard band at the $X$ point the presence of a lower energy feature (see the Supp. Material [26]). The strength of the occupied part of the spectra $(\omega<0)$ has been enhanced by a factor of 4 to enable a better visualization. For reference, we also include the standard LDA calculation in solid black lines [23. Right panel: The total local $\operatorname{DOS}(\omega)$. The (red) arrows indicate the main contributions that should be observed in optical conductivity. The (black) arrow indicates the small transport gap $\Delta$, consistent with the value measured in resistivity experiments [15].

due to the strong orbital polarization of the LDA bands at the $X, Y$, and $Z$ points of the Brillouin Zone (see the Supplemental Material [26]). The detailed study of this point and the calculation of the optical response are beyond the scope of the present study. Finally, we should also mention that the small gap in the $\operatorname{DOS}(\omega)$ of magnitude $\Delta \approx 0.30 \mathrm{eV}$ is also consistent with the value 0.241 $\mathrm{eV}$ reported from the activated behavior of the resistivity at ambient pressure [13.

To conclude, we presented a combined experimental and theoretical study of the Mott-Hubbard insulatorto-metal transition in the strongly correlated system $\mathrm{GaTa}_{4} \mathrm{Se}_{8}$. Our resistivity experiments under quasihydrostatic pressure on GTS single crystals enable us to uncover the first-order character of the correlation-driven paramagnetic 3D Mott transition. Moreover, we achieved control of a resistive bistability at the transition, providing validation of its physical nature, as was qualitatively predicted by DMFT more than 20 years ago. We have provided further and decisive support by means of GTS specific LDA+DMFT calculations, which are in good agreement with the present and recently reported experiments.

We thank V. Ta Phuoc, V. Guiot, and P. Stoliar for useful discussions. We acknowledge support from the French Agence Nationale de la Recherche (ANR-09Blan-0154-01) and (R.W. and C.A.) from CONICET and ANPCyT (PIP 114-201101-00376, PICT-2012-0609, and PIP 112-201101-00536). 
* On leave from Departamento de Física, FCEN, UBA and IFIBA, CONICET

[1] M. Imada, A. Fujimori and Y. Tokura, Rev. Mod. Phys. 70, 1039 (1998).

[2] A. Georges, G. Kotliar, W. Krauth M. J. Rozenberg, Rev. Mod. Phys 6813 (1996).

[3] D. B. Mc Whan and J. P. Remeika, Phys. Rev. B 2, 3734 (1970).

[4] M. J. Rozenberg et al., Phys. Rev. Lett. 75, 105 (1995).

[5] P. Limelette et al., Science 302, 8992 (2003).

[6] K. Held, G. Keller, V. Eyert, D. Vollhardt and V.I. Anisimov, Phys. Rev. Lett. 86, 5345 (2001).

[7] P. Hansmann et al., Phys. Status Solidi B 250, No. 7, 1251 (2013).

[8] L. Paolasini et al., Phys. Rev. Lett. 82, 4719 (1999).

[9] G. Kotliar et al., Rev. Mod. Phys. 78, 865 (2006).

[10] M. M. Abd-Elmeguid et al., Phys. Rev. Lett. 93126403 (2004).

[11] R. Pocha, D. Johrendt R. Pöttgen, Chem. Mater. 122882 (2000).

[12] R. Pocha, D. Johrendt, B. Ni M. M. Abd-Elmeguid, J. Am. Chem. Soc. 1278732 (2005).

[13] V. Guiot, E. Janod, B. Corraze and L. Cario, Chem. Mat. 23, 2611 (2011).

[14] L. Cario, C. Vaju, B. Corraze, V. Guiot and E. Janod, Adv. Mater. 22, 5193 (2010).

[15] V. Ta Phuoc et al., Phys. Rev. Lett. 110, 037401 (2013).

[16] E. Dorolti et al., J. Amer. Chem. Soc. 132, 5704 (2010).

[17] C. Vaju, et al., Adv. Mat. 202760 (2008).

[18] V. Guiot et al, Nat.Comm. 4, 1722 (2013).

[19] P. Stoliar et al., Adv. Mat. 25, 3222 (2013).

[20] H. Barz, Mater. Res. Bull. 8983 (1973).
[21] C. Perrin, R. Chevrel M. C. Sergent, R. Acad. Sci. Ser. C 280949 (1975).

[22] H. Müller, W. Kockelmann, D. Johrendt, Chem. Mat. 18 2174 (2006).

[23] A. Camjayi, R. Weht and M. J. Rozenberg, Eur. Phys. Lett. 100, 57004 (2012).

[24] D. Johrendt, Z. Anorg. Allg. Chem. 624952 (1998).

[25] V. I. Anisimov, F. Aryasetiawan A. I. Lichtenstein, J. Phys.: Condens. Matter 9767 (1997).

[26] See Supplemental Material, which includes Refs. 27+35 for technical details of experiments and calculations.

[27] P. Blaha, K. Schwarz, G. K. H. Madsen, D. Kvasnicka and J. Luitz, An Augmented Plane Wave + Local Orbitals Program for Calculating Crystal Properties (Karlheinz Schwarz, Techn. Universität Wien, Austria, 2001).

[28] D. J. Singh and L. Nordstrom, Planewaves, Pseudopotentials and the LAPW Method (Springer: New York, 2006).

[29] J. P. Perdew and Y. Wang, Phys.Rev. B 4513244 (1992).

[30] N. Marzari and D. Vanderbilt, Phys. Rev. B 5612847 (1997); N. Marzari, A. A. Mostofi, J. R. Yates, I. Souza, and D. Vanderbilt, Rev. Mod. Phys. 841419 (2012).

[31] A. A. Mostofi, J. R. Yates, Y.-S. Lee, I. Souza, D. Vanderbilt and N. Marzari, Comput. Phys. Commun. 178 685 (2008).

[32] J. Kunes, R. Arita, P. Wissgott, A. Toschi, H. Ikeda and K. Held, Comp. Phys. Commun. 1811888 (2010).

[33] E. Gull, A. J. Millis, A. I. Lichtenstein, A. N. Rubtsov, M. Troyer and P. Werner, Rev. Mod. Phys. 83349 (2011).

[34] K. Haule, Phys. Rev. B 75155113 (2007).

[35] V. S. Oudovenko, G. Pálsson, K. Haule, G. Kotliar and S. Y. Savrasov, Phys. Rev. B 73, 035120 (2006).

[36] M. J. Rozenberg, Phys. Rev. B 55, R4855 (1997). 


\section{Supplemental Material of "First-order insulator to metal Mott-transition in the paramagnetic $3 \mathrm{D}$ system $\mathrm{GaTa}_{4} \mathrm{Se}_{8}$ "}

\section{EXPERIMENTAL DETAILS}

The high pressure was applied using a quasihydrostatic experimental setup, corresponding to a Bridgman configuration with WC anvils, where pyrophillite is used as a gasket and steatite as the pressure medium that favors quasi-hydrostatic conditions. Pressure inside the cell was determined by measuring the resistive superconducting transition of $\mathrm{Pb}$. The pressure gradient was estimated from the width of this transition and corresponds to a $5-10 \%$ of the applied pressure. A standard 4 terminal DC technique was used to measure resistivity under high pressure at different temperatures. The typical size of the samples was of a fraction of a $\mathrm{mm}$. Electrical contacts were made using $25 \mu \mathrm{m}$ diameter Pt wires pressed to the sample's surface by the pressure setup. A well calibrated carbon-glass thermometer thermally anchored to the anvils ensures a determination of sample's temperature with an uncertainty lower than $0.5 \mathrm{~K}$ for the whole temperature range studied.

\section{NUMERICAL CALCULATION DETAILS}

\section{LDA}

In the LDA calculation we use the Wien2K code 1, that is an implementation of the full-potential linearizedaugmented plane wave method (FP-LAPW) 2]. As we are not interested in total energies nor magnetic states, we adopt the simplest local density approximation to represent the exchange-correlation potential [3]. Results using the generalized gradient approximation are completely equivalent since band structures are, in general, insensitive to this choice. Even thought within the DFT schemes the eigenvalues and eigenfunctions formally have no direct physical meaning, it is, nevertheless, broadly accepted that they provide a good approximation for the quasi-particle energies and band-structure. Thus, here we shall adopt them as the reference for the calculation of the localized Wannier orbital basis 4. These Wannier orbitals represent the molecular orbitals arising from the $\mathrm{Ta}_{4}$ clusters, which are well defined as the distances between the four metal atoms in the cluster are significantly shorter than the inter-cluster ones. We restrict the energy window to the three $t_{2 g}$ bands that cross the Fermi energy. We note that these three bands are quite well separated from the rest of the band manifold, which is very advantageous in order to successfully obtain an accurate local orbital basis representation with short ranged hopping amplitudes. The localized Wannier orbital basis is computed following the procedure described by Marzari, Vanderbilt and coworkers in a series of papers [5] and implemented in the code Wannier90 6]. As the interface between the two programs we use the code wien2wannier [7.

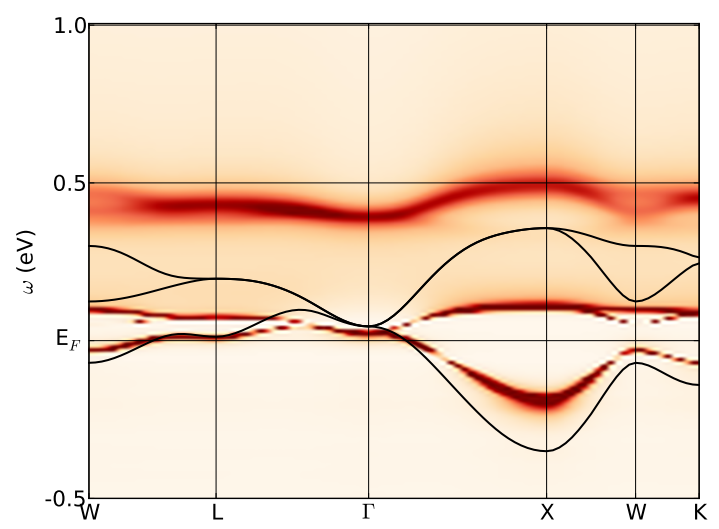

FIG. S1. Intensity plot of the electronic structure of the $t_{2 g}$ bands of GTS obtained from LDA+DMFT calculations for $U$ $=1.2 \mathrm{eV}$ and $T=58 \mathrm{~K}$ within the coexistence region. The data correspond to the metallic solution that coexists with the insulator one, shown in Fig. 4 of the main text. For reference, we also include the standard LDA calculation in solid black lines [4].

\section{DMFT}

The DMFT calculation is done using a continuoustime quantum Monte Carlo (CTQMC) impurity solver [8], based on the hybridization expansion [9]. In order to perform reliable analytic continuation of the imaginary axis data a large number of Monte Carlo steps has to be adopted to minimize statistical errors. Special care has been taken to obtain well converged solutions in the coexistence region, which is complicated by critical slowing down and enhanced statistical fluctuations. Due to the general cubic symmetry of the system, the doublecounting problem is absent.

The correlation effects to the LDA bandstructure is obtained via a DMFT self-energy, which is calculated from an auxiliary quantum-impurity problem. The numerical calculations are done with a multi-orbital CTQMC method, which allows us to reach the relevant temperature regime. To explore experimentally the IMT, external pressure was applied, which affects the (bare) bandwidth $W$ and, hence, the strength of electronic correlations $U / W$. In principle, one could simulate this by recomputing the LDA bandstructure at the compressed 
atomic positions. This methodologically demanding and full empirical information is not available, thus, we shall proceed in a different simpler manner. From previous LDA calculation under pressure [10, it is possible to see that the bandstructure is modified by a simple scale factor in the range of pressures considered experimentally, without changing qualitatively the dispersion relation. Since we choose the same value of $U$ for all interorbital interactions, we shall keep the ambient pressure LDA electronic structure fixed and use $U$ as the free parameter to change the $U / W$ ratio.

\section{LDA+DMFT metallic solution}

For completeness, we show in Fig. S1 the LDA+DMFT results for the bandstructure of the correlated metallic solution at $U=1.2 \mathrm{eV}$ and $T=58 \mathrm{~K}$. The electronic structure near the Fermi energy shows a narrowing with respect to the LDA bands, which reflects the mass renormalization of the correlated metallic state. In addition, at higher energies one finds spectral weight associated to the Hubbard bands. The lower Hubbard band cannot be observed in the intensity plot due to its relatively weak spectral strength.

\section{Resistivity}

The calculation of the resistivity is done following the definition [11]: $\rho=\frac{k_{B} T}{e^{2}} \frac{1}{A_{0}}$ with

$$
A_{0}=\lim _{\omega \rightarrow 0}\left[\frac{\hbar}{\omega_{n} \beta} \int_{0}^{\beta} \mathrm{d} \tau e^{i \omega_{n} \tau}\left\langle\left\langle j^{x}(\tau) j^{x}(0)\right\rangle\right\rangle\right]_{\omega_{n} \rightarrow \omega+i 0^{+}}
$$

We evaluate the correlation function $\left\langle\left\langle j^{x}(\tau) j^{x}(0)\right\rangle\right\rangle$ within DMFT, in the dipole approximation and ignoring vertex corrections.

\section{ORBITAL POLARIZATION IN K-SPACE}

The LDA bandstructure in the maximally localized Wannier orbital representation is the starting point for the LDA+DMFT calculation. We adopt the construction made in Ref. 4. In Fig. S2, we reproduce the bandstructure of the three $t_{2 g}$ fatbands, which indicate the orbital character across the Brillouin zone.

As expected, at the $\Gamma$-point the three bands are degenerate, as dictated by the cubic lattice symmetry. We also observe that from the $\Gamma$ - to the $L$-point the orbital character of the three bands is almost degenerate. Interestingly, however, the situation is dramatically different from the $\Gamma$ - to the $X$-point. In this case we observe that at the $X$-point the character of the band beneath the

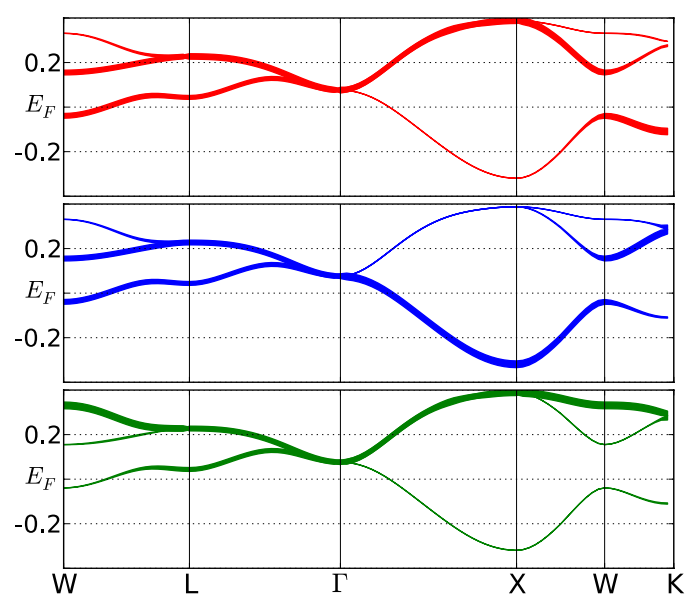

FIG. S2. LDA calculated "Fat bands" of GTS (bottom). Red, blue and green respectively correspond to $d_{x y}, d_{y z}$ and $d_{z x}$ characters.

Fermi level is dominated by the $y z$ orbital. Similarly, by symmetry, we have $z x$ at the $Y$-point and $x y$ at the $Z$-point. Hence, while the system is degenerate at the $\Gamma$-point, there is a strong orbital polarization at the $X$, $Y$ and $Z$-points. This feature, which we shall explore in detail elsewhere is responsible for the small band that is observed at low (positive) energies at the $X$ point of the upper Hubbard band, in Fig. 4 of the main text.

* On leave from Departamento de Física, FCEN, UBA and IFIBA, CONICET

[1] P. Blaha, K. Schwarz, G. K. H. Madsen, D. Kvasnicka and J. Luitz, An Augmented Plane Wave + Local Orbitals Program for Calculating Crystal Properties (Karlheinz Schwarz, Techn. Universität Wien, Austria, 2001).

[2] D. J. Singh and L. Nordstrom, Planewaves, Pseudopotentials and the LAPW Method (Springer: New York, 2006).

[3] J. P. Perdew and Y. Wang, Phys.Rev. B 4513244 (1992).

[4] A. Camjayi, R. Weht and M. J. Rozenberg, Eur. Phys. Lett. 100, 57004 (2012).

[5] N. Marzari and D. Vanderbilt, Phys. Rev. B 5612847 (1997); N. Marzari, A. A. Mostofi, J. R. Yates, I. Souza, and D. Vanderbilt, Rev. Mod. Phys. 841419 (2012).

[6] A. A. Mostofi, J. R. Yates, Y.-S. Lee, I. Souza, D. Vanderbilt and N. Marzari, Comput. Phys. Commun. 178 685 (2008).

[7] J. Kunes, R. Arita, P. Wissgott, A. Toschi, H. Ikeda and K. Held, Comp. Phys. Commun. 1811888 (2010).

[8] E. Gull, A. J. Millis, A. I. Lichtenstein, A. N. Rubtsov, M. Troyer and P. Werner, Rev. Mod. Phys. 83349 (2011).

[9] K. Haule, Phys. Rev. B 75155113 (2007).

[10] V. Ta Phuoc et al., Phys. Rev. Lett. 110, 037401 (2013).

[11] V. S. Oudovenko, G. Pálsson, K. Haule, G. Kotliar and S. Y. Savrasov, Phys. Rev. B 73, 035120 (2006). 